\title{
EXPERIMENTAL AND NUMERICAL STUDY ON PDMS COLLAPSE FOR FABRICATION OF MICRO/NANOCHANNELS
}

\author{
Zhifu Yin * Helin Zou ${ }^{* *}$
}

\begin{abstract}
PDMS (polydimethylsiloxane) collapse method is a simple and low cost approach for micronanochannel fabrication. However, the bonding pressure which influences the size of the final PDMS micro/nanochannels has not yet been studied. In this study, the effect of the bonding pressure on the size and maximum local stress of the PDMS micronanochannels was investigated by both experimental and numerical simulation method. The results show that when the bonding pressure is lower than 0.15 MPa the experiment results can agree well with the simulation results. The fluorescent images demonstrate that there is no blocking or leakage over the entire micro/nanochannels.
\end{abstract}

K e y w o r d s: PDMS collapse, micro/nanochannel, bonding, numerical simulation

\section{INTRODUCTION}

Micro/nanochannels have become a fundamental and critical technique to study fluidic and ion properties because of the special phenomena which only occurred in micro/nanochannels $[1,2]$. Nowadays, a substantial amount of research has been reported such as ionic transport $[3,4]$, protein concentration [5], virus characterization [6] and DNA analysis $[7,8]$.

To fabricate micro/nanochannels, many techniques have been proposed. The most commonly used method is beam based methods. By using electron beam [9-11], proton beam [12, 13], or focused ion beam $[14,15]$, silicon or quartz micro/nanochannels can be easily fabricated. However, this technique can not widely accessible, since the equipments these methods used are very expensive. Especially, for large-scale fabrication of micro/nanochannels, they have extremely high costs. Deposition and dry etching method is a relative low cost method [16-18]. Based on this method, silicon, glass, or quartz micro/nanochannels can be fabricated [19]. However, the cost of the micro/nanochannels fabricated by this method is still high, because it requires reactive ion etching, deep reactive ion etching or metal deposition technique. To decrease the cost of the micro/nanochannels, the plastic micro/nanochannels fabrication techniques, such as thermal nanoimprinting and ultraviolet nanoimprinting technique, were proposed [20, 21]. This well developed technique has great potential to fabricate plastic micro/nanochannels with low cost, large scale and high throughput. However, most of the commercially thermoplastic materials used in nanoimprinting suffer from a high auto-fluorescence when excited by ultraviolet radiation $[22,23]$. Auto-fluorescence background interferes with on-chip optical detection, which making plastic micro/nano devices usually very difficult to measure the fluorescent molecules in the channels. PDMS is a low auto-fluorescence material, which can be widely used in the fluorescent detecting experiments [24, 25]. Therefore, PDMS becomes an ideal material to fabricate micro/nanochannels with low auto-fluorescence. PDMS micro/nanochannels can be easily fabricated by PDMS casting method [26, 27]. However, to fabricate PDMS nanochannels by casting method, convex silicon or glass nano-mold should be fabricated. And it is difficult to fabricate silicon or glass nano-mold with low cost. In recent years, PDMS collapse method was proposed [2830]. By this simple method PDMS micro/nanochannels can be fabricated with extremely low cost in only two steps (ie PDMS microchannels casting step and PDMS bonding step). In contrast to the these state-of-the-art micro/nanochannel fabrication methods, PDMS collapse method shows considerably some advantages including low-cost, mass production, less consumption of harmful chemicals, and ease of operation in micro/nanofabrication procedures. PDMS bonding process is a step in which the PDMS microchannels were highly deformed under bonding pressure and thus nanochannels were formed at the bottom of the microchannels. This process is critical and has great impact on the final size of the PDMS micro/nanochannels. Unfortunately, there is no work which would analyze the PDMS deformation process. An indepth and systematic study on the PDMS deformation process should be made to investigate the deformation of the PDMS substrate during the bonding process.

In this paper, numerical simulation on the deformation of the PDMS substrate during the bonding process was carried out based on Mooney-Rivlin model. The influence of the bonding pressure on the width and depth of the micro/nanochannel was studied. And the effect of the bonding pressure on the max local stress in the PDMS

School of Mechanical Science and Engineering, Jilin University, Changchun 130012, China, yinzf@jlu.edu.cn; ${ }^{* *}$ Key Laboratory for Micro/Nano Technology and Systems of Liaoning Province, Dalian University of Technology, Dalian 116024, China 
substrate was investigated. To verify the proposed simulation method, numerical simulation results and experimental results were compared. To characterize the fabricated nanochannels, fluorescent dye test was conducted.

\section{THEORETICAL MODEL FOR ANALYSIS OF PDMS COLLAPSE PROCESS}

PDMS is a hyper-elastic material. At mixing ratio of 15:1, the PDMS can be elongated as high as $200 \%$ under break load of about 0.9 MPa [31]. During PDMS collapse process, PDMS exhibits its hyper-elastic property. We assume that PDMS is incompressible and isotropic. Its mechanical properties can be represented by MooneyRivlin model. The Mooney-Rivlin strain energy function can be expressed as [32]

$$
W-C_{10}\left(I_{1}-3\right)+C_{01}\left(I_{2}-3\right),
$$

where, $C_{10}, C_{01}$ are physical constants characterizing the PDMS, and $I_{1}, I_{2}$ are strain invariants.

As Hocheng reported [33], the physical constants C10, C01 can be evaluated by Youngs modulus of PDMS

$$
C_{01}=0.25 C_{10}, \quad 6\left(C_{10}-C_{01}\right) \approx E
$$

where, $E$ is the Youngs modulus of the PDMS.

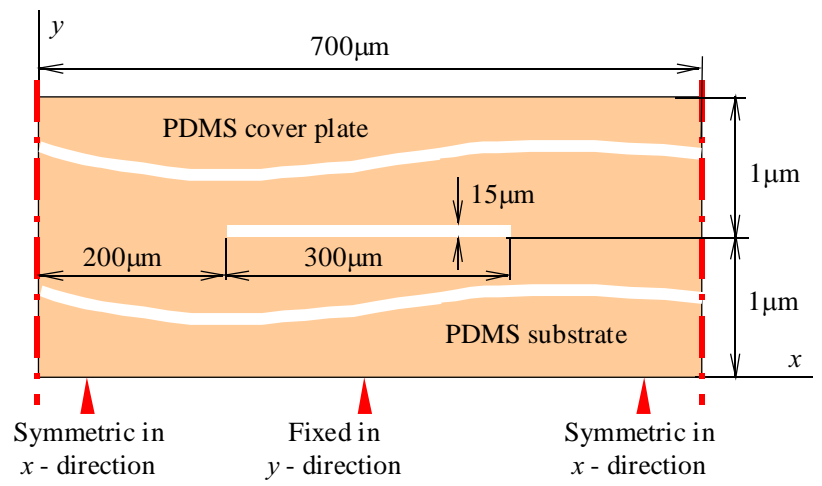

Fig. 1. The model used for numerical simulation

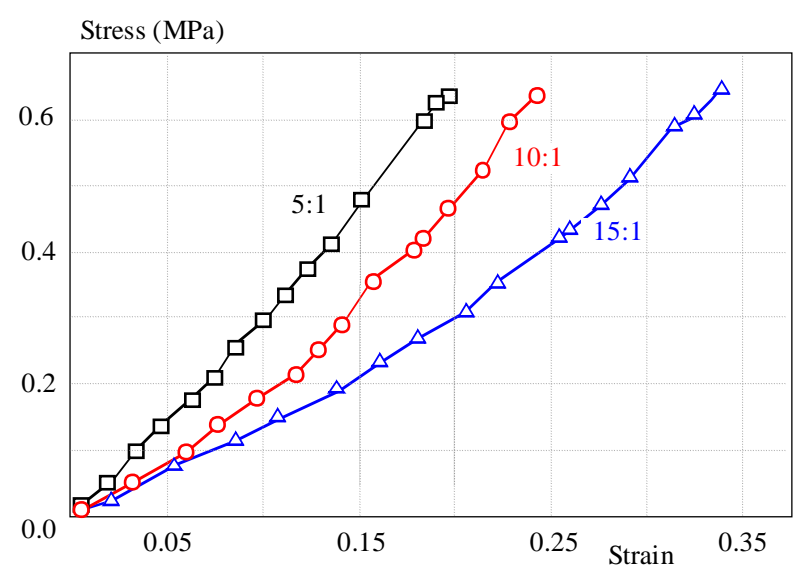

Fig. 2. PDMS strain-stress curves with different mixing ratio
To analyze the deformation of the PDMS during collapse process, the commercial finite element analysis software, ANSYS 10.0 (ANSYS, Pennsylvania, USA), is employed during numerical simulation. The geometric model is shown in Fig. 1. For simplification, the 2D (two dimensional) plain-strain condition is assumed. Since the PDMS cover plate has periodical structures, only one periodical feature was modeled to the finite element domain. Symmetric boundaries were applied on both sides of the PDMS cover plate and the PDMS substrate. The Y-axis displacement of the bottom surface of the PDMS substrate was set to zero since the PDMS substrate was supported by a fixed glass board.

\section{EXPERIMENTS}

As discussed above, to calculate the parameters of the Mooney-Rivlin model, the Young modulus of the PDMS should be measured. The Young modulus is not a constant value which can ranges from several mega Pascal to dozens of mega Pascal $[34,35]$. It varies with the mixing ratio of base polymer to curing agent, vacuum pressure, and curing temperature [34]. The Young modulus depends on the PDMS fabrication process. Therefore, in this study the strain-stress curves were measured to obtain the Young modulus of the PDMS.

A commercial PDMS material (Sylgard 184, Dow Corning, Michigan, USA) was used to fabricate bulk PDMS. The bulk PDMS with mixing ratio of 5:1, 10:1, and 15:1 (Volume/Volume) were fabricated. Firstly, the PDMS and the curing agent was pipetted by a syringe and then thoroughly mixed. The mixture was degassed for the first time at $10 \mathrm{~Pa}$ for $20 \mathrm{~min}$ to eliminate the trapped air bubble. Then the mixture was poured onto a PMMA container. The container is a cube with edge length of $2 \mathrm{~cm}$. The mixture was degassed at $10 \mathrm{~Pa}$ for $20 \mathrm{~min}$ for the second time to eliminate the air bubble again. Then the PMMA container was placed in the leveled baking oven and the mixture was cured at $50 \mathrm{C}$ for $4 \mathrm{~h}$. After the mixture was totally cured, the cube PDMS was fabricated.

To measure the Young modulus of the PDMS, hot embossing equipment made by Dalian University of Technology was used. The hot embossing equipment consists of a forcing system, a heating/cooling system and a monitoring/controlling system. During the hot embossing process, the forcing system provides necessary compression force with an accuracy of $0.5 \mathrm{~N}$. The force and displacement can be dynamically controlled and recorded by the monitoring/ controlling system. Fig. 2 shows the strainstress curves of the PDMS with mixing ratio of 5:1, 10:1, and 15:1. There is a nearly linear relationship between the strain and the stress. Fitted by the linear function in the OriginPro 8 (OriginLab Corp. Northampton, USA), the 

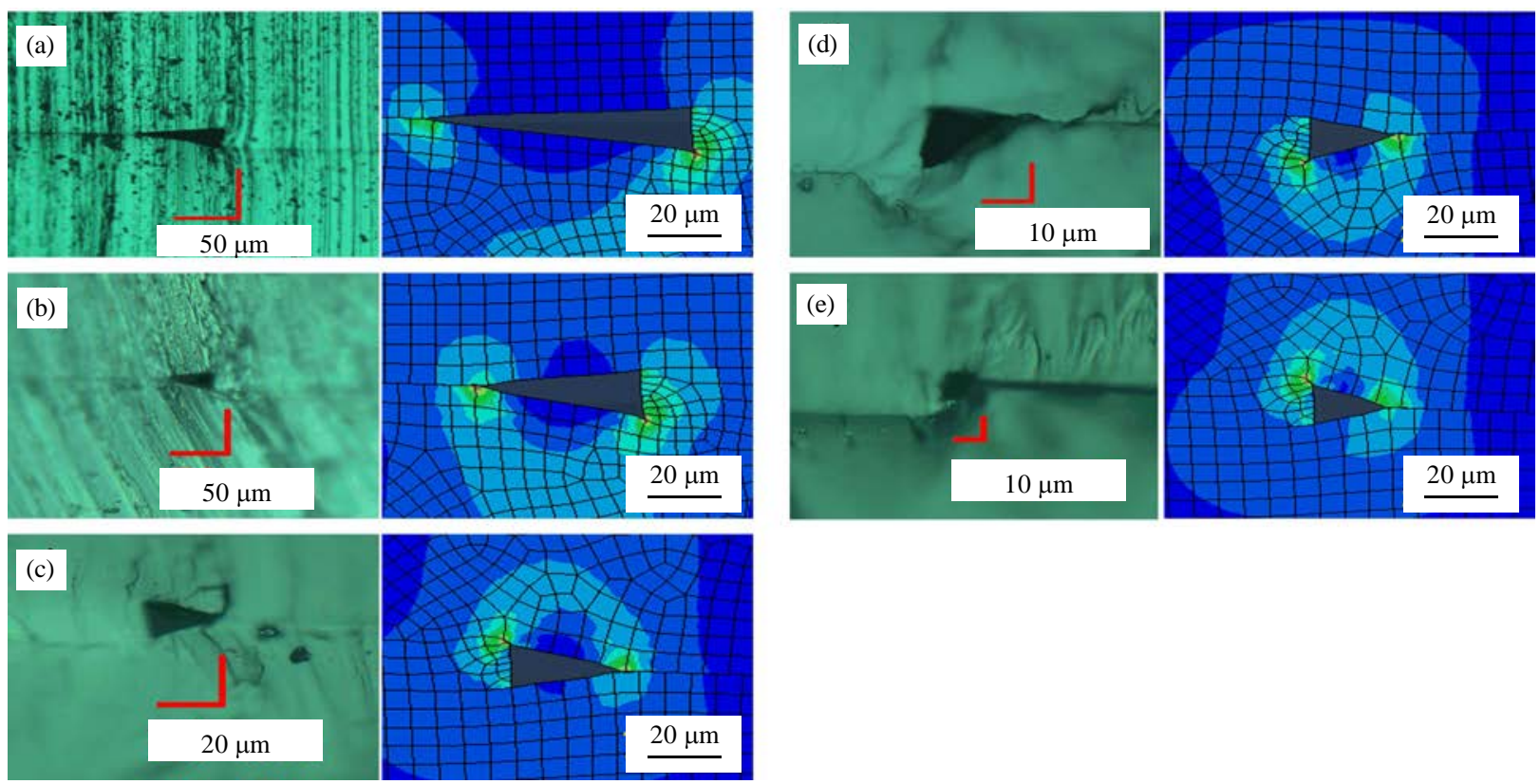

Fig. 3. Experimental and simulation comparison of the collapsed PDMS profiles bonded at pressure of: (a) - 0.05 MPa, (b) - 0.10 $\mathrm{MPa},(\mathrm{c})-0.15 \mathrm{MPa},(\mathrm{d})-0.20 \mathrm{MPa}$, and (e) - $0.25 \mathrm{MPa}$
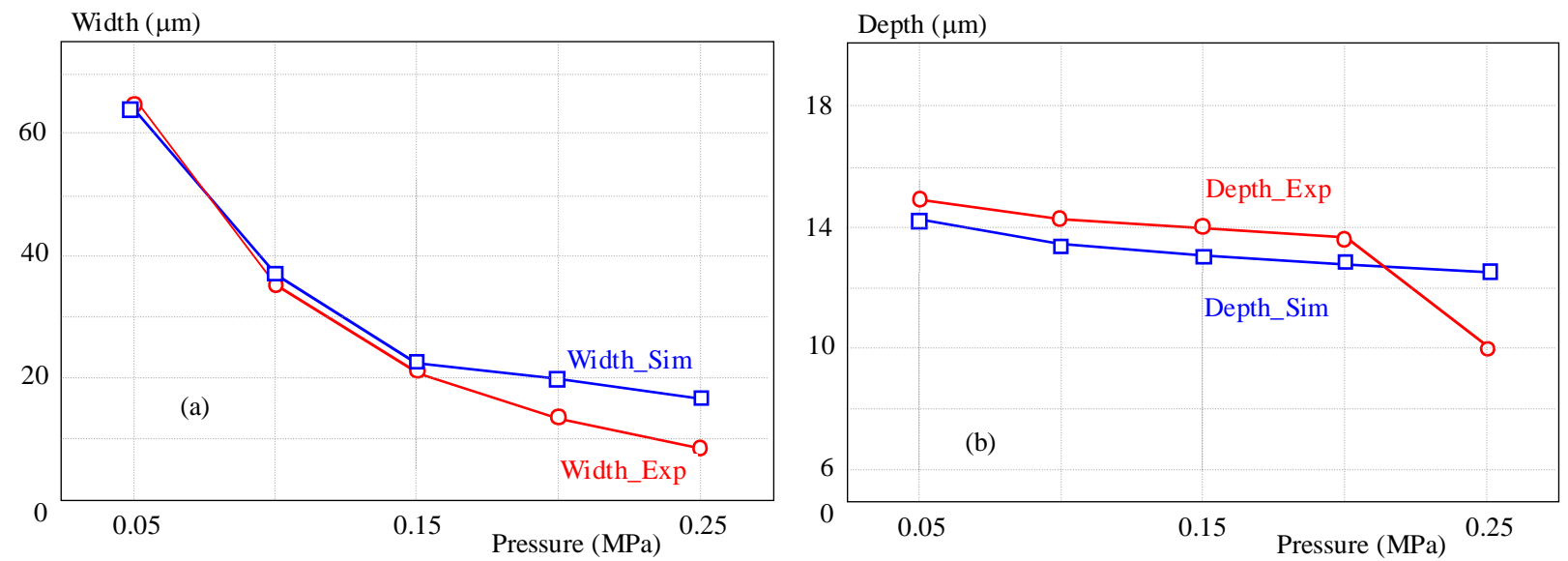

Fig. 4. Comparison of the (a) — width and (b) - depth of the microchannels between experiment and simulation results

Young modulus of the PDMS can be calculated. The average correlation of curve fitting at different mixing ratio is as high as $\mathrm{R} 2=98.3 \%$. The fitting results show that the Young modulus is $3.27 \mathrm{MPa}$ for the PDMS with mixing ratio of 5:1, 2.61 $\mathrm{MPa}$ for mixing ratio of $10: 1$, and 1.90 $\mathrm{MPa}$ for mixing ratio of 15:1.

\section{RESULTS AND DISCUSSION}

\subsection{PDMS microchannels fabrication}

To verify the proposed simulation method, PDMS microchannels were fabricated by PDMS collapse method. To fabricate PDMS microchannels by PDMS collapse, the PDMS mesas (width of $300 \mu \mathrm{m}$ and depth of $15 \mu \mathrm{m}$ were fabricated into PDMS cover plate by PDMS casting. The mixing ratio of PDMS cover plate and PDMS substrate was 10:1. The deformation of the PDMS cover plate during PDMS collapse was analyzed by both experiment and numerical simulation. During PDMS microchannels fabrication, different bonding pressures were applied which ranges from $0.05 \mathrm{MPa}$ to $0.25 \mathrm{MPa}$.

Figure 3 shows the comparisons of the collapsed PDMS profiles analyzed by experiment and numerical simulation methods. The left images (green images) are experiment results, while the right images (blue images) are numerical simulation results. It can be seen that the widths and depths of the PDMS decreased with the increase of the bonding pressures.

To investigate the influence of the bonding pressure on the dimension of the PDMS microchannels, the widths and depths of the PDMS microchannels were measured. The measuring results are shown in Fig. 4 from which, one can see that the width of the microchannels can decrease 
significantly with the increase of the bonding pressure. However, the depth of the microchannels can only decrease slightly with the increase of the bonding pressure. Comparing the experiment results (red line) with the numerical simulation results (black line) (Fig. 4 a), the experiment results can agree well with the numerical simulation results, when the bonding pressure was smaller than 0.15 MPa. When the bonding pressure was larger than $0.15 \mathrm{MPa}$, the experiment results cannot agree with the numerical simulation results. That is because when applied high bonding pressure (larger than $0.15 \mathrm{MPa}$ ), the PDMS can be seriously deformed and the contact area cannot be well controlled. Thus, the widths of the microchannels analyzed by experiments did not agree with that analyzed by numerical simulation. From Fig. 4b), the experiment depths were larger than the simulation ones.

During numerical simulation, the sidewall of the PDMS mesa (the red line in Fig. 5) is assumed to be perpendicular to the surface of the PDMS mesa. After bonding, the depth of the PDMS microchannel is $d_{2}$. However, in the experiments, the sidewall (the blue dot line in Fig. 5) may not be perpendicular to the surface of the PDMS mesa. The angle ? between PDMS mesa and PDMS sidewall is larger than $90{ }^{\circ} \mathrm{C}$ (as shown in Fig. 5). During bonding process, the contact area between the sidewall and the surface of the PDMS mesa is smaller than that in numerical simulation. After bonding, the depth of the PDMS microchannel $d_{1}$ (experiment) is thus larger than $d_{2}$ (simulation). In order to investigate the dimension of the PDMS microchannels during bonding process, the bonding pressure is suggested to be smaller than 0.15 $\mathrm{MPa}$. In this way, the dimension analyzed by numerical simulation can agree well with the one analyzed by experiment.

When the PDMS cover plate collapse, the cover plate will bonded with the substrate. After releasing the bonding pressure, the PDMS cover plate cannot be recovered. The stress will be generated between the bonded interfaces. The maximum local stress in the PDMS is an important factor to evaluate the fabrication quality of the PDMS microchanels. When the maximum local stress is larger than the breaking strength of the PDMS, the PDMS will be broken. The microchannels can thus be damaged. To ensure the PDMS microchannels intact, the maximum local stress should be smaller than the breaking strength of the PDMS. By numerical simulation, the stress in the PDMS can be easily investigated. Fig. 6 shows the maximum local stress in the PDMS microchannels bonded at different pressures. The maximum local stress increases with the increase of the bonding pressure. Since with higher bonding pressure, the PDMS cover plate can be deformed more seriously, and the dimension of the microchannel will be smaller. After releasing the bonding pressure, the deformation of the PDMS cover plate cannot be recovered because the cover plate was bonded with the substrate. Therefore, higher stress is generated in the PDMS mirochannels.

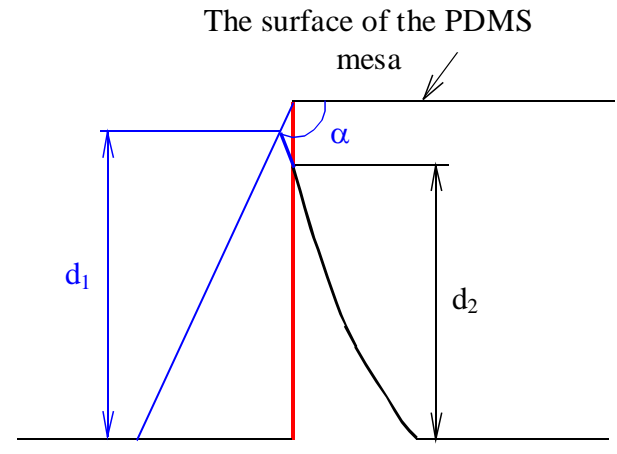

Fig. 5. The reason of experiment widths larger than the simulation ones

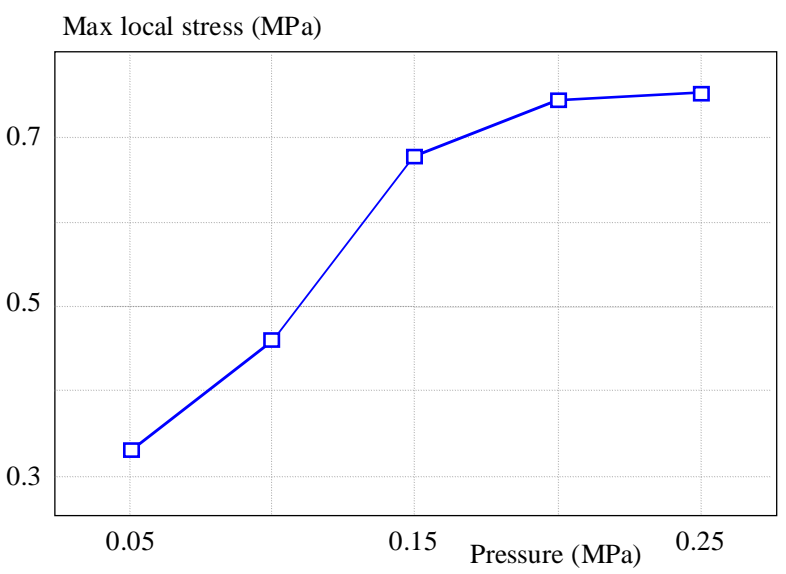

Fig. 6. The max local stress in the PDMS microchannels bonded at different pressures

\subsection{PDMS nanochannels fabrication}

To fabricate PDMS nanochannels by PDMS collapse, the PDMS mesas (width of $300 \mathrm{~m}$ and depth of $100 \mathrm{~nm}$ ) and PDMS mesas (width of $300 \mathrm{~m}$ and depth of $200 \mathrm{~nm}$ ) were fabricated into PDMS cover plate by PDMS casting method. The mixing ratio of PDMS cover plate and PDMS substrate was 10:1. The deformation of the PDMS cover plate during PDMS collapse was analyzed by numerical simulation. During PDMS nanochannels fabrication, different bonding pressures were applied on the cover plate ranging from $0.05 \mathrm{MPa}$ to $0.25 \mathrm{MPa}$.

Figure 7(a) and Fig. 8(a) show the width and depth of the nanochannels after PDMS collapse under different bonding pressure ranging from 0.05 $\mathrm{MPa}$ to $0.25 \mathrm{MPa}$. The width and depth of the nanochannels decreased with the increase of the bonding pressure. From bonding pressure of $0.05 \mathrm{MPa}$ to bonding pressure of $0.15 \mathrm{MPa}$, the width and depth of the nanochannel decreased significantly. However, from bonding pressure of $0.15 \mathrm{MPa}$ to bonding pressure of $0.25 \mathrm{MPa}$, the width and depth of the nanochannel decreased slightly. Fig. 7(b) and Fig. 8(b) show the max local stress in the PDMS nanochannels after the release of the bonding pressure. The max local stress increased with the increase of the bonding pressure. According the discussion above, one can see that 

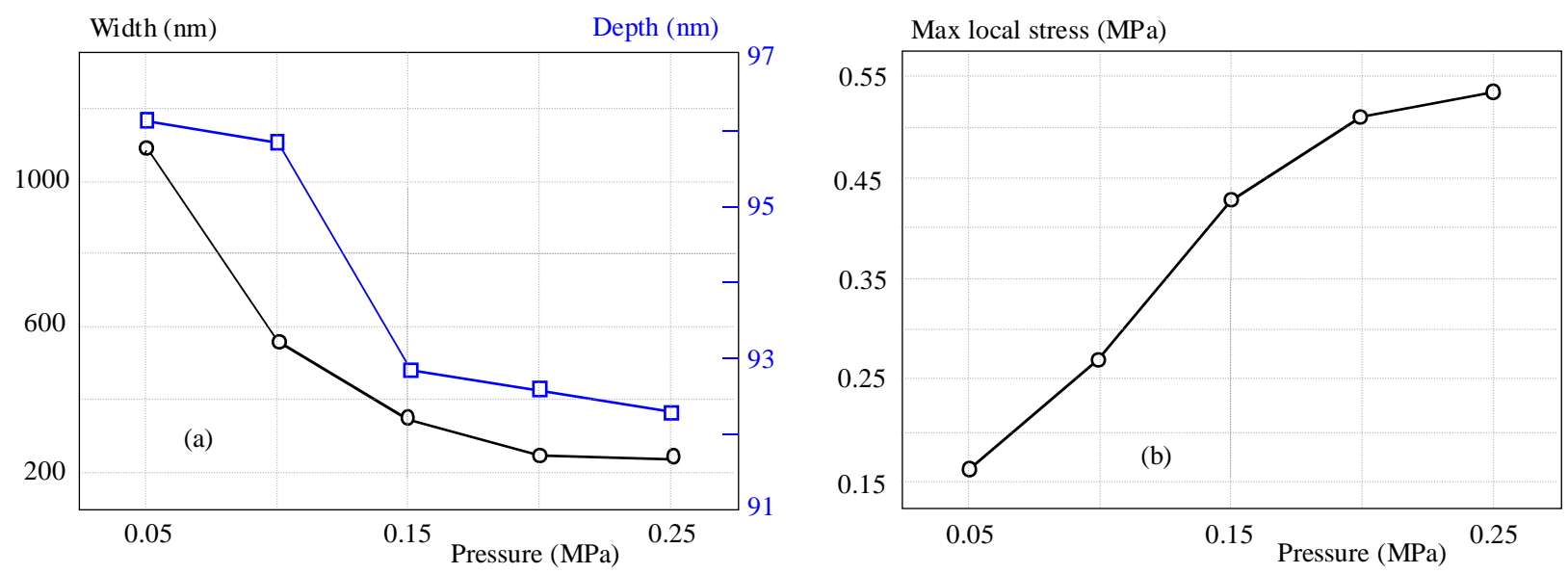

Fig. 7. The size and of the max local stress of the PDMS nanochannels (original depth of $100 \mathrm{~nm}$ ), (a) — the width and depth of the PDMS nanochannels bonded at pressures ranging from 0.05 MPa to $0.25 \mathrm{MPa}$, and (b) - the max local stress in the PDMS nanochannels bonded at pressures ranging from $0.05 \mathrm{MPa}$ to $0.25 \mathrm{MPa}$.
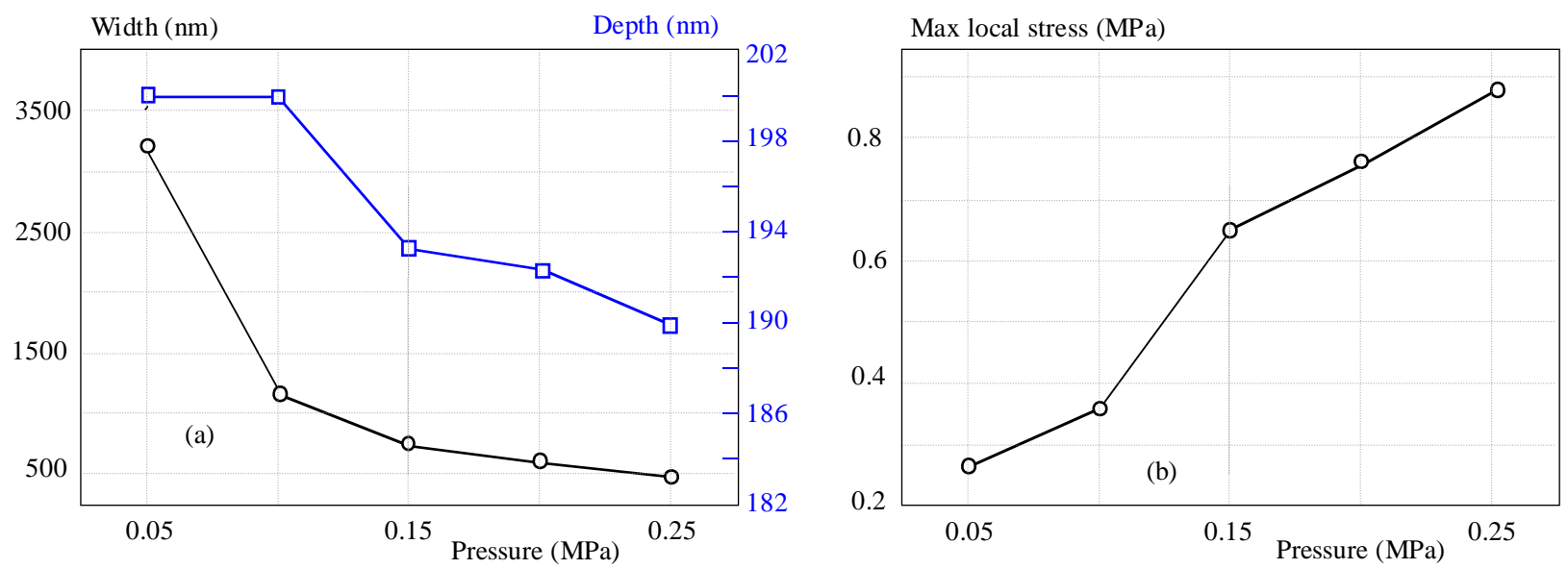

Fig. 8. The size and of the max local stress of the PDMS nanochannels (original depth of $200 \mathrm{~nm}$ ), (a) — the width and depth of the PDMS nanochannels bonded at pressures ranging from $0.05 \mathrm{MPa}$ to $0.25 \mathrm{MPa}$, and (b) - the max local stress in the PDMS nanochannels bonded at pressures ranging from $0.05 \mathrm{MPa}$ to $0.25 \mathrm{MPa}$.

high bonding pressure can lead to small nanochannels. However, high bonding pressure can also result in high local stress. To fabricate small nanochannels with moderate local stress, during PDMS collapse bonding pressure of $0.15 \mathrm{MPa}$ is suggested.

It is difficult to obtain a SEM image of the bonded PDMS nanochannels, because during cutting off the PDMS, the PDMS can deform significantly and the PDMS nanochannels can deform seriously. It is hard to observe an intact PDMS nanochannel under scanning electron microscope. To characterize the bonding PDMS nanochannels, we filled fluorescent dye into the nanochannels, and then we observed fluorescent dye in the nanochannels. Fig. 9 shows the microscope image of the bonded PDMS nanochannels filled by fluorescent dye. The microscope image demonstrates that there is no leakage or block in the bonded PDMS nanocannels. The nanochannels were bonded at bonding pressure of 0.15 $\mathrm{MPa}$. The width of the nanochannels was about $800 \mathrm{~nm}$, while the depth of the nanochannels was about $193 \mathrm{~nm}$.

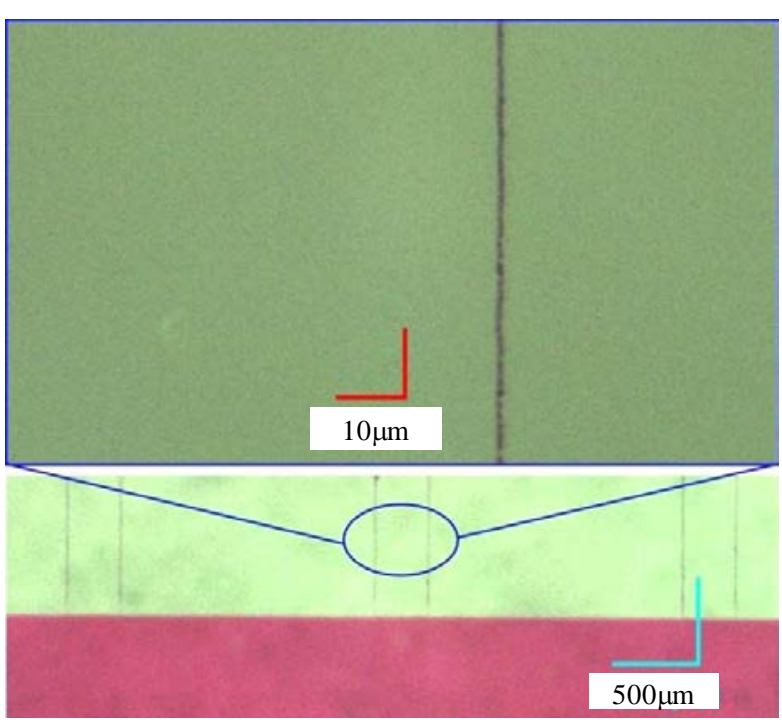

Fig. 9. The microscope image of the bonded PDMS nanochannels filled by fluorescent dye 


\section{CONCLUSION}

In the present paper, a micro/nanochannels fabrication method by PDMS collapse was proposed. The bonding process was analyzed by both numerical and experimental method. During numerical simulation, MooneyRivlin model was used. The influence of the bonding pressure on the width and depth of the micro/nanochannel was studied. And the effect of the bonding pressure on the max local stress in the PDMS substrate was also investigated. It indicated that the simulation results were in good agreement with experimental results. The fluorescent dye test demonstrates that the absence of leakage and block in the bonded PDMS nanochannels. It is expected that the proposed simulation method have a high potential for the analysis of PDMS bonding process.

\section{Acknowledgement}

This project is supported by Specialized Research Fund for the Doctoral Program of Higher Education of China (SRFDP) (No. 20120041110034).

\section{REFERENCES}

[1] SPARREBoOM, W.-VANDENBERG, A.-EIJKEL, J. C. T. : Principles and applications of nanofluidic transport, Nature Nanotechnology 4 No. 11 (2009), 713-720.

[2] FREEDMAN, K. J.-HAQ, S. R.-EDEL, J. B.-JEMTH, P.-KIM, M. J.: Single molecule unfolding and stretching of protein domains inside a solid-state nanopore by electric field, Scientific Reports 3 (2013), 1638.

[3] SCHOCH, R. B.-RENAUD, P.: Ion transport through nanoslits dominated by the effective surface charge, Applied Physics Letters 86 No. 25 (2005), 253111.

[4] PLECIS, A.-SCHOCH, R. B.-RENAUD, P.: Ionic transport phenomena in nanofluidics, Nano Letters, 5 No. 6 (2005), 1147-1155.

[5] WANG, Y. C.-STEVEnS, A. L.-HAN, J. Y.: Million-fold preconcentration of proteins and peptides by nanofluidic filter, Analytical Chemistry, 77 No. 14 (2005), 4293-4299.

[6] ZHOU, K.-LI, L.-TAN, Z.-ZLOTNICK, A.-JACOBSON, S. C. : Characterization of Hepatitis B Virus Capsids by Resistive-Pulse Sensing, Journal of the American Chemical Society, 133 No. 6 (2011), 1618-1621.

[7] FU, J. P.-MAO, P.-HAN, J. Y.: Nanofilter array chip for fast gel-free biomolecule separation, Applied Physics Letters, 87 No. 26 (2005), 263902.

[8] ABGRALL, P.-LOW, L.-N.-NGUYEN, N.-T.: Fabrication of planar nanofluidic channels in a thermoplastic by hot-embossing and thermal bonding, Lab on a Chip, 7 No. 4 (2007), 520-522.

[9] CHOI, S.-YAN, M.-ADESIDA, I. : Fabrication of triangular nanochannels using the collapse of hydrogen silsesquioxane resists, Applied Physics Letters, 93 No. 16 (2008), 163113.

[10] YASUI, T.-KAJI, N.-OGAWA, R.-HASHIOKA, S.-TOKESHI, M.-HORIIKE, Y.-BABA, Y.: DNA separation in nanowall array chips, Analytical Chemistry, 83 No. 17 (2011), 6635-6640.

[11] NAM, S. W.-LEE, M. H.-LEE, S. H.-LEE, D. J.-ROSSNAGEL, S. M.-KIM, K. B.: Sub-10-nm nanochannels by self-sealing and self-limiting atomic layer deposition, NanoLetters, 10 No. 9 (2010), 3324-3329.
[12] FAN, L.-KHENGBOON, T.-MALAR, P.-BIKKAROLLA, S. K.-VANKAN, J. A. : Fabrication of nickel molds using proton beam writing for micro/nanofluidic devices, Microelectronic Engineering, 102 (2012), 36-39.

[13] VANKAN, J. A.-ZHANG, C.-MALAR, P. P.-van der MAAREL, J. R. C.: High throughput fabrication of disposable nanofluidic lab-on-chip devices for single molecule studies, Biomicrofluidics, 6 No. 3 (2012), 036502.

[14] MENARD, L. D.-RAMSEY, J. M.: Fabrication of sub-5 nm nanochannels in insulating substrates using focused ion beam milling, NanoLetters, 11 No. 2 (2011), 512-517.

15] FANZIO, P.-MUSSI, V.-MANNESCHI, C.-ANGELI, E.FIRPO, G.-REPETTOL.-VALBUSA, U.: DNA detection with a polymeric nanochannel device, Lab on a Chip, 11 No. 17 (2011), 2961-2966.

[16] PHAN, V. N.-NGUYEN, N. T.-YANG, C.-JOSEPH, P.GUE, A. M.: Fabrication and experimental characterization of nanochannels, Journal of Heat Transfer, 134 No. 5 (2012), 051012 .

[17] RIEHN, R.-AUSTIN, R. H.-STURM, J. C.: A nanofluidic railroad switch for DNA, NanoLetters, 6 No. 9 (2006), 1973-1976.

18] KUTCHOUKOV, V. G.-LAUGERE, F.-VANDERVLIST, W.-PAKULA, L.-GARINI, Y.-BOSSCHE, A.: Fabrication of nanofluidic devices using glass-to-glass anodic bonding, Sensors and Actuators a-Physical, 114 No. 2-3 (2004), 521-527.

[19] MAO, P.-HAN, J. Y.: Fabrication and characterization of 20 $\mathrm{nm}$ planar nanofluidic channels by glass-glass and glass-silicon bonding, Lab on a Chip, 5 No. 8 (2005), 837-844.

[20] CHANTIWAS, R.-HUPERT, M. L.-PULLAGURLA, S. R.BALAMURUGAN, S.-TAMARIT-LOPEZ, J.-PARK, S.DATTA, P.-GOETTERT, J.-CHO, Y.-K.-SOPER, S. A. : Simple replication methods for producing nanoslits in thermoplastics and the transport dynamics of double-stranded DNA through these slits, Lab on a Chip, 10.

[21] TENG, L.-KIRCHNER, R.-PLOETNER, M.-TUERKE, A.-JAHN, A.-HE, J.-HAGEMANN, F.-FISCHER, W.-J. : Fabrication and characterization of sub-500 nm channel organic field effect transistor using UV nanoimprint lithography with cheap Si-mold, Microelectronic Engineering, 97 (2012), 38-42.

[22] HAWKINS, K. R.-YAGER, P.: Nonlinear decrease of background fluorescence in polymer thin-films - a survey of materials and how they can complicate fluorescence detection in mu TAS, Lab on a Chip, 3 No. 4 (2003), 248-252.

[23] LLOPIS, S. D.-STRYJEWSKI, W.-SOPER, S. A. : Near-infrared time-resolved fluorescence lifetime determinations in poly(methylmethacrylate) microchip electrophoresis devices, Electrophoresis, 25 No. 21-22 (2004), 3810-3819.

[24] XU, B. Y.-XU, J. J.-XIA, X. H.-CHEN, H. Y. : Large scale lithography-free nanochannel array on polystyrene, Lab on a Chip, 10 No. 21 (2010), 2894-2901.

[25] LO, K. F.-JUANG, Y. J.: Fabrication of long poly(dimethyl siloxane) nanochannels by replicating protein deposit from confined solution evaporation, Biomicrofluidics, 6 No. 2 (2012), 026504 .

26] KIM, S. H.-CUI, Y.-LEE, M. J.-NAM, S.-W.-OH, D.KANG, S. H.-KIM, Y. S.-PARK, S.: Simple fabrication of hydrophilic nanochannels using the chemical bonding between activated ultrathin PDMS layer and cover glass by oxygen plasma, Lab on a Chip, 11 No. 2 (2011), 348-353.

[27] JOHN, H.: The molding of biological features using a flexible polymer mold, Micron, 42 No. 5 (2011), 429-433.

[28] KIM, B.-HEO, J.-KWON, H. J.-CHO, VS. J.-HAN, J.-KIM, S. J.-LIM, G. : Tunable Ionic Transport for a Triangular Nanochannel in a Polymeric Nanofluidic System, Acs Nano, 7 No. 1 (2013), 740-747.

29] LEE, J.-YOON, Y.-K.-KIM, J.-KIM, Y.-JO, K.: Roofcollapsed PDMS mask for nanochannel fabrication, Bulletin of the Korean Chemical Society, 32 No. 1 (2010), 33-34. 
[30] PARK, S. M.-HUH, Y. S.-CRAIGHEAD, H. G.-ERICKSON, D.: A method for nanofluidic device prototyping using elastomeric collapse, Proceedings of the National Academy of Sciences of the United States of America, 106 No. 37 (2009), 15549-15554.

[31] TAEKYUNG, K.-JEONGKOO, K.-OKCHAN, J. : Measurement of nonlinear mechanical properties of PDMS elastomer, Microelectronic Engineering, 88 No. 8 (2011), 1982-5.

[32] NAGARAJAN, P.-YAO, D. : Uniform Shell Patterning Using Rubber-Assisted Hot Embossing Process. II. Process Analysis, Polymer Engineering and Science, 51 No. 3 (2011), 601-608.

[33] HOCHENG, H.-NIEN, C. C. : Numerical analysis of effects of mold features and contact friction on cavity filling in the nanoimprinting process, Journal of Microlithography Microfabrication and Microsystems 5 No. 1 (2006), 011004.

[34] XIANGDONG, Y.-HONGZHONG, L.-YUCHENG, D. : Research on the cast molding process for high quality PDMS molds, Microelectronic Engineering, 86 No. 3 (2009), 310-13.

[35] MYEOngsub, K.-BYEONG-UI, M.-HIDROvO, C. H. : Enhancement of the Thermo-mechanical Properties of PDMS Molds for the hot Embossing of PMMA Microfluidic Devices, Journal of Micromechanics and Microengineering, 23 No. 9 (2013), 095024.
Received 1 September 2016

Zhifu Yin is a lecturer at Jilin University in China. He obtained PhD degree in Dalian University of Technology, China, in 2016. His research interests lie in the fabrication and application of micro- and nano-devices used for biological, medical and mechanical fields. More than 20 international journal and conference papers have been published based on the above researches.

Helin Zou is a professor at Dalian University of Technology in China. He obtained PhD degree in University of Wales, Cardiff, UK, in 2001, responsible for courses and his research interests lie in the application of microfabrication technology to the development of micro-engineered systems aimed at providing an information-gathering front end to electronic system. More than 50 international journal and conference papers have been published based on the above researches. He is member of the British Institute of Physics and Micro/Nano society of China. 